\title{
Fetal Hemodynamic Response to Maternal Isometric Exercise
}

\section{Karina Biaggio Soares*, Francisco Maximiliano Pancich Gallarreta, Walter Santos Neme}

Federal University of Santa Maria, Santa Maria, Rio Grande do Sul, Brazil

Email: *karinabiaggiosoares@icloud.com

How to cite this paper: Soares, K.B., Gallarreta, F.M.P. and Neme, W.S. (2018) Fetal Hemodynamic Response to Maternal Isometric Exercise. Open Journal of Obstetrics and Gynecology, 8, 541-552. https://doi.org/10.4236/ojog.2018.86061

Received: March 19, 2018

Accepted: June 1, 2018

Published: June 4, 2018

Copyright ( 92018 by authors and Scientific Research Publishing Inc. This work is licensed under the Creative Commons Attribution International License (CC BY 4.0).

http://creativecommons.org/licenses/by/4.0/

\section{cc) (i) Open Access}

\begin{abstract}
Background: Studies on physical activity during pregnancy and its impact on mother and fetus are still limited. International protocols consider only aerobic exercise and fail to provide information about other modalities such as isometric exercise. Isometric exercise promotes cardiorespiratory resistance and muscle strengthening, but it is rarely tested on pregnant women because it increases maternal blood pressure and can subsequently affect placental circulation. Objective: To evaluate maternal and fetal response of low-risk pregnant women undergoing isometric testing through a Doppler velocimetry study. Methodology: A cross-sectional, experimental study was performed on 46 healthy pregnant women (gestational age between 26 and 36 weeks) who underwent isometric testing. This testing was performed using a handgrip dynamometer to measure maternal and fetal hemodynamic parameters before, during, and after isometric testing. Results: There was a significant increase in systolic blood pressure (BP; pre-isometrics $113.13 \pm 9.92 \mathrm{mmHg}$, during isometrics $117.13 \pm 10.24 \mathrm{mmHg}$, and post-isometrics $112.43 \pm 9.87$ $\mathrm{mmHg}, \mathrm{p}<0.001)$ and heart rate (HR; pre-isometrics $87.52 \pm 14.10 \mathrm{bpm}$, during isometrics $97.61 \pm 14.83 \mathrm{bpm}$, and post-isometrics $85.13 \pm 13.24 \mathrm{bpm}$, $\mathrm{p}<0.001$ ). There were significant decreases in the pulsatility index (PI; pre-isometrics $0.63 \pm 0.15$, during isometrics $0.56 \pm 0.15$, and post-isometrics $0.65 \pm 0.17, \mathrm{p}=0.001$ ), resistance index (RI; pre-isometrics $0.44 \pm 0.08$, during isometrics $0.40 \pm 0.07$, and post-isometrics $0.45 \pm 0.08, \mathrm{p}=0.001$ ), and systolic/diastolic (S/D) ratio (pre-isometrics $1.81 \pm 0.26$, during isometrics $1.69 \pm 0.24$, and post-isometrics $1.85 \pm 0.29, \mathrm{p}<0.001)$ of the left uterine artery (UA). These results showed significant changes only during the isometric exercise, and not between the pre- and post-isometric exercises. There were no significant differences in fetal parameters when the results before, during, and after the isometric test were compared. Conclusion: Isometric testing had no repercussions for fetal hemodynamics in healthy low-risk pregnant women.
\end{abstract}




\section{Keywords}

Isometric Testing, Doppler Velocimetry, Fetal Hemodynamics, Maternal Hemodynamics

\section{Introduction}

Physical activity is fundamental in all stages of life. Its health benefits are proven and include an improved cardiorespiratory system and lower risks of obesity and other comorbidities associated with a sedentary lifestyle [1] [2]. For women during pregnancy and the perinatal period, exercise helps control maternal weight gain, prevents the onset of gestational diabetes and preeclampsia, accelerates postpartum recovery, and reduces cesarean delivery rates, all without additional risks to the pregnancy or the fetus [3] [4].

However, studies on physical activity during pregnancy and its impact on mother and fetus, particularly in terms of different modalities and types of physical training, are still limited [3] [4].

International protocols [1] [2] consider only aerobic exercise and fail to provide information about other modalities such as isometric exercise. This modality is rarely tested on pregnant women because it increases maternal blood pressure and can subsequently affect placental circulation [5].

Isometric exercise, which involves muscular contraction without changes in movement, is a part of daily activities, such as lifting and carrying heavy objects. By promoting cardiorespiratory resistance and muscle strengthening, isometric exercise aids in the prevention of lower back pain and contributes to the quality of other types of exercises, including aerobic exercise [5] [6] [7].

Therefore, the objective of this study was to evaluate maternal and fetal response of low-risk pregnant women undergoing isometric testing through a Doppler velocimetry study.

\section{Material and Methods}

\section{Study design}

A cross-sectional study was performed on healthy pregnant women receiving prenatal care from public health care centers in Santa Maria, Rio Grande do Sul State, Brazil. The study was performed from September to December 2017, on women at a gestational age between 26 and 36 weeks due to proximity of delivery. The sample calculation for significance of $5 \%$ and test power of $80 \%$, based on a previous meta-analysis [7], indicated a sample of 50 healthy pregnant women. All subjects signed an informed consent form provided by the researchers.

The exclusion criteria were history of previously diagnosed chronic disease (such as arterial hypertension or diabetes mellitus), smoking habit, drinking habit, use of illicit drugs, complications diagnosed prior to data collection (such as 
preterm labor, premature rupture of membranes, placenta previa, multiple gestation, and gestational diabetes), diagnosis of preeclampsia at any time during pregnancy, and any of the contraindications to the practice of physical activity provided by the 2015 ACOG guidelines (Table 1) [2].

Pregnant women from eight primary public health care centers in Santa Maria, Rio Grande do Sul State, received written invitations to participate in the study voluntarily during their prenatal visits. Telephone calls were made to 60 pregnant women to explain the research protocol and to schedule the data collection, which was performed in the Department of Fetal Medicine of the Santa Maria University Hospital (HUSM). The data collection was done after the study was approved by the institution's Research Ethics Committee.

Upon arrival, the women were instructed on how to use the manual handgrip dynamometer (Crown manual dynamometer with $100 \mathrm{kgf}$ capacity), and they performed three consecutive attempts at maximum voluntary contraction (MVC). The mean score of these three attempts was the MVC used for data collection. Next, the women remained at rest in the semi-Fowler position for $10 \mathrm{~min}$.

Maternal pre-isometric data were then collected, including blood pressure, or BP (using a Welch Allyn ${ }^{\mathrm{mm}}$ manual blood pressure cuff), heart rate (HR), and uterine artery (UA) Doppler ultrasound results (using a GE Voluson i portable ultrasound machine with a $3.5-5.0 \mathrm{MHz}$ convex transducer). The fetal pre-isometric data collected were HR and the Doppler results for the umbilical artery (UmA), medial cerebral artery (MCA), and ductus venosus (DV). The Doppler study variables used were pulsatility index (PI), resistance index (RI), and the systolic/diastolic (S/D) ratio.

Table 1. Contraindications to aerobic exercise during pregnancy.

\begin{tabular}{ll}
\hline \multicolumn{1}{c}{ Absolute } & \multicolumn{1}{c}{ Relative } \\
\hline -Hemodynamically significant heart disease & -Anemia \\
-Restrictive lung disease & -Unevaluated maternal cardiac arryhthmia \\
-Incompetent cervix or cerclage & -Chronic bronchitis \\
-Multiple gestation at risk of premature labor & -Poorly controlled type 1 diabetes \\
-Persistent second- or third-trimester bleeding & -Extreme morbid obesity \\
-Placenta previa after 26 weeks of gestation & -Extreme underweight \\
-Premature labor during the current pregnancy & -History of extremely sedentary lifestyle \\
-Rupture of membranes & -Intrauterine growth restriction in the \\
-Preeclampsia or pregnancy-induced hypertension & current pregnancy \\
-Severe anemia & -Poorly controlled hypertension \\
& -Orthopedic limitations \\
& -Poorly controlled seizure disorder \\
& -Poorly controlled hyperthyroidism \\
& -Heavy smoking
\end{tabular}

Source: adapted from ACOG [2], PM = Premature labor; IGR = Intrauterine growth restriction. 
After their data at rest were collected, the women began the isometric tests using the manual dynamometer with a contraction corresponding to $50 \%$ of MVC. Isometric positions were held for at least $1 \mathrm{~min}$ after the Doppler was initiated, and they were sustained until $4-7$ waves considered adequate by the examiner were captured. This step was repeated at least five times for the collection of the five variables of the Doppler study, with a 1-min interval between contractions. If fetal movements prevented the collection of fetal variables, the women were instructed to repeat the isometric test after the usual 1-min interval. During the isometric contractions, all maternal and fetal data were collected again, always after $1 \mathrm{~min}$ holding the isometric contraction.

At the end of the isometric test, the subjects rested again for $5 \mathrm{~min}$, during which fetal biometry was performed, amniotic fluid was quantified, and the placenta was evaluated. All maternal and fetal post-isometric variables were then collected again.

All data from the Doppler study, including the calculation results of the Doppler variables, and on fetal biometry were obtained using the GE Voluson i ultrasound machine and the 4c-RS convex transducer provided by the HUSM Department of Fetal Medicine. These steps were performed by the most experienced researcher, who performed all of the collections.

\section{Data analysis}

Statistical analyses were performed with Statistical Package for the Social Sciences software, version 21, using Friedman's two-way analysis of variance by ranks and a significance level of $\mathrm{p}<0.05$. The Bonferroni correction was then applied to compare the data sets from the collection (from the pre-isometric, isometric, and post-isometric periods).

\section{Results}

Of the 60 pregnant women scheduled, four did not attend the visit, three were excluded because of technical difficulties in performing the Doppler study, two were excluded due to suspected preeclampsia at the time of collection (these two were referred for consultation in the emergency department of the hospital, where the diagnosis was confirmed), and one woman was excluded for being a smoker, resulting in a total of 50 pregnant women as subjects.

In the collection of the postnatal data, four subjects were found to have developed preeclampsia at the end of pregnancy and were therefore excluded from the statistical analyses (Figure 1).

The mean age of the subjects was $25.69( \pm 6.90)$ years (range: 15 - 43 years). In terms of parity, $24(52.17 \%)$ were nulliparous and $22(47.83 \%)$ were multiparous. The mean gestational age (GA) at the time of sample collection was 33.22 $( \pm 2.05)$ weeks (range from 28 weeks and 5 days to 36 weeks). The rate of sedentary lifestyles among the subjects was $76.08 \%$ before pregnancy and $91.30 \%$ during pregnancy. The prevalence of active pregnant women was $8.7 \%$ (Table 2).

At the beginning of the research protocol, the subjects were asked about their 


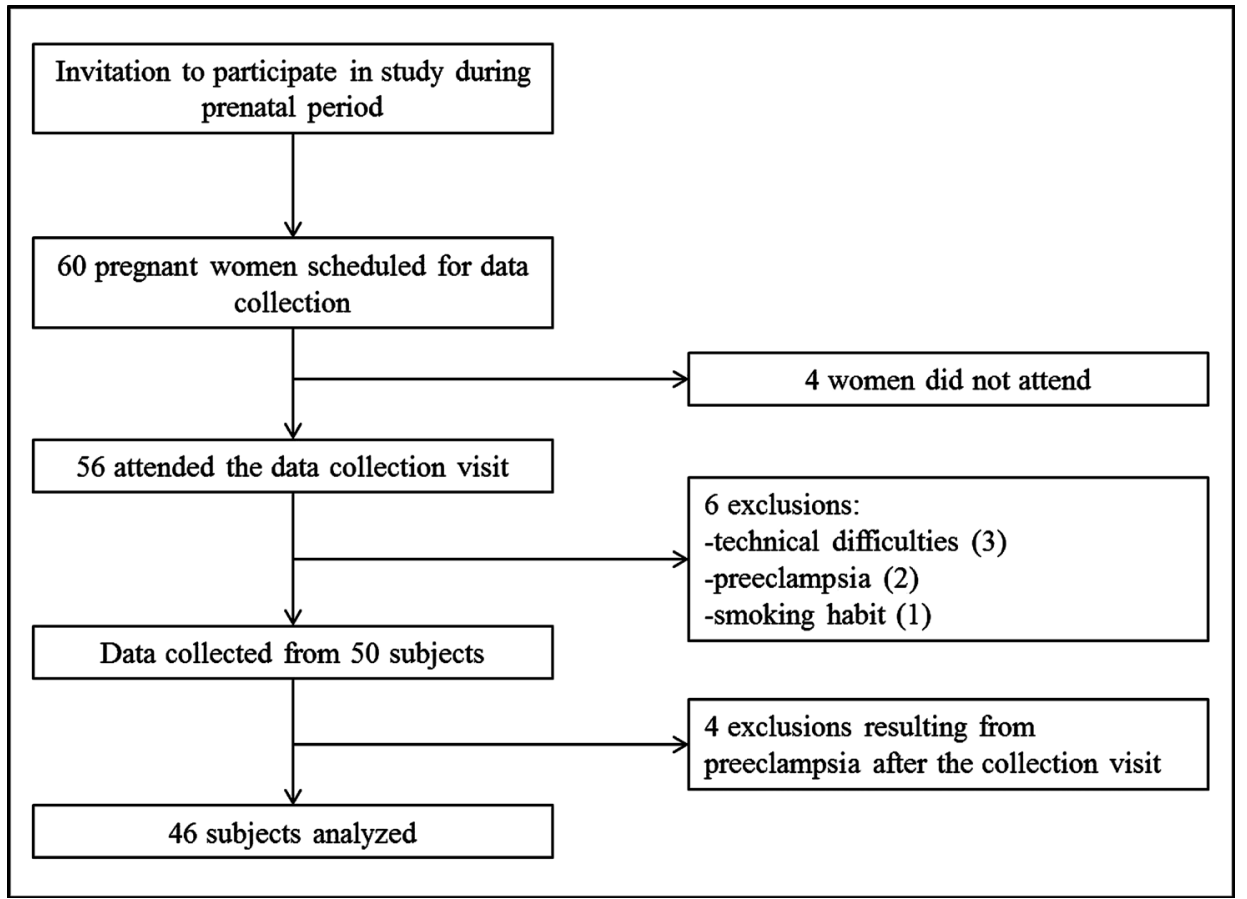

Figure 1. Protocol flowchart.

Table 2. Description of the sample of pregnant women.

\begin{tabular}{cc}
\hline Maternal age & Total $(\mathrm{N}=46)$ \\
Parity & $25.69( \pm 6.90)$ \\
Nulliparous & $24(52.17 \%)$ \\
Multiparous & $22(47.83 \%)$ \\
Gestational age (weeks) & $33.22( \pm 2.05)$ \\
Sedentary before pregnancy? & \\
Yes & $35(76.08 \%)$ \\
No & $11(23.91 \%)$ \\
Sedentary during pregnancy? & $42(91.30 \%)$ \\
Yes & $4(8.70 \%)$ \\
No & \\
Dominant hand & $40(86.96 \%)$ \\
Right & $6(13.04 \%)$ \\
Left &
\end{tabular}

Data represent mean \pm standard deviation or number of cases with their percentages.

dominant hand, which was most commonly the right hand (86.96\%). They were also asked to perform an MCV on the hand grip dynamometer using the dominant hand. The mean MVC was 21.22 ( \pm 3.90$) \mathrm{kgf}$ (range: 15 - $32 \mathrm{kgf}$ ).

The analysis of the results of maternal parameters showed that there was a 
significant increase $(\mathrm{p}<0.001)$ in systolic BP (mean pre-isometric value of $113.13 \pm 9.92 \mathrm{mmHg}$, during isometrics $117.13 \pm 10.24 \mathrm{mmHg}$, and post-isometrics $112.43 \pm 9.87 \mathrm{mmHg}$ ) and HR (pre-isometrics $87.52 \pm 14.10 \mathrm{bpm}$, during isometrics $97.61 \pm 14.83 \mathrm{bpm}$, and post-isometrics $85.13 \pm 13.24 \mathrm{bpm}$ ). There were significant decreases in the PI (pre-isometrics $0.63 \pm 0.15$, during isometrics 0.56 \pm 0.15 , and post-isometrics $0.65 \pm 0.17 ; \mathrm{p}=0.001$ ), RI (pre-isometrics $0.44 \pm$ 0.08 , during isometrics $0.40 \pm 0.07$, and post-isometrics $0.45 \pm 0.08 ; \mathrm{p}=0.001$ ), and S/D ratio (pre-isometrics $1.81 \pm 0.26$, during isometrics $1.69 \pm 0.24$, and post-isometrics $1.85 \pm 0.29 ; \mathrm{p}<0.001$ ) of the left UA (Table 3). These results differed significantly only during isometric exercise, and not between pre- and post-isometric exercises. The right UA exhibited similar behavior, but the decreases in PI, RI, and S/D ratio were not significant.

There were no significant differences in fetal parameters when the results before, during, and after the isometric test were compared.

Table 3. Analysis of maternal and fetal pre-isometric, during isometric and post-isometric variables.

\begin{tabular}{|c|c|c|c|c|c|c|c|}
\hline \multicolumn{8}{|c|}{ post-hoc Bonferroni } \\
\hline & Pre & During & Post & $\mathrm{p}$ & $\mathrm{P} 1$ & $\mathrm{P} 2$ & P3 \\
\hline SBP & $113.13( \pm 9.92)$ & $117.13( \pm 10.24)$ & $112.43( \pm 9.87)$ & 0.000 & 0.004 & 0.004 & 1.000 \\
\hline DBP & $72.83( \pm 7.86)$ & $73.43( \pm 9.16)$ & $73.83( \pm 7.09)$ & 0.685 & - & - & - \\
\hline MHC & $87.52( \pm 14.10)$ & $97.61( \pm 14.83)$ & $85.13( \pm 13.24)$ & 0.000 & 0.000 & 0.000 & 0.526 \\
\hline PI RUA & $0.64( \pm 0.17)$ & $0.62( \pm 0.17)$ & $0.66( \pm 0.19)$ & 0.139 & - & - & - \\
\hline RI RUA & $0.44( \pm 0.08)$ & $0.43( \pm 0.08)$ & $0.45( \pm 0.08)$ & 0.112 & - & - & - \\
\hline SD RUA & $1.83( \pm 0.27)$ & $1.79( \pm 0.29)$ & $1.86( \pm 0.31)$ & 0.089 & - & - & - \\
\hline PI LUA & $0.63( \pm 0.15)$ & $0.56( \pm 0.15)$ & $0.65( \pm 0.17)$ & 0.001 & 0.012 & 0.002 & 1.000 \\
\hline RI LUA & $0.44( \pm 0.08)$ & $0.40( \pm 0.07)$ & $0.45( \pm 0.08)$ & 0.001 & 0.007 & 0.002 & 1.000 \\
\hline SD LUA & $1.81( \pm 0.26)$ & $1.69( \pm 0.24)$ & $1.85( \pm 0.29)$ & 0.000 & 0.003 & 0.001 & 1.000 \\
\hline FHR & $139.54( \pm 11.76)$ & $141.09( \pm 11.30)$ & $139.71( \pm 9.61)$ & 0.559 & - & - & - \\
\hline MCA PI & $1.81( \pm 0.35)$ & $1.87( \pm 0.37)$ & $1.83( \pm 0.36)$ & 0.729 & - & - & - \\
\hline MCA RI & $0.82( \pm 0.06)$ & $0.82( \pm 0.06)$ & $0.82( \pm 0.06)$ & 0.927 & - & - & - \\
\hline MCA SD & $6.24( \pm 2.24)$ & $6.57( \pm 2.39)$ & $6.32( \pm 2.04)$ & 0.816 & - & - & - \\
\hline UmA PI & $0.92( \pm 0.16)$ & $0.94( \pm 0.17)$ & $0.94( \pm 0.18)$ & 0.856 & - & - & - \\
\hline UmA RI & $0.61( \pm 0.07)$ & $0.61( \pm 0.07)$ & $0.61( \pm 0.08)$ & 0.994 & - & - & - \\
\hline UmA SD & $2.64( \pm 0.50)$ & $2.66( \pm 0.57)$ & $2.70( \pm 0.60)$ & 0.983 & - & - & - \\
\hline DV PI & $0.60( \pm 0.23)$ & $0.58( \pm 0.17)$ & $0.60( \pm 0.17)$ & 0.872 & - & - & - \\
\hline
\end{tabular}

Values represent mean \pm standard deviation. P: Friedman's two-way analysis of variance of ranks. P1: Bonferroni correction comparing pre-isometric and during isometric results; P2: Bonferroni correction comparing during isometric and post-isometric results; P3: Bonferroni correction comparing pre-isometric and post-isometric results. SBP: systolic blood pressure, in $\mathrm{mmHg}$; DBP: diastolic blood pressure, in $\mathrm{mmHg}$; MHR: maternal heart rate, in beats per minute (bpm); PI: pulsatility index; RI: resistance index; SD: systolic/diastolic ratio; RUA: right uterine artery; LUA: left uterine artery; FHR: fetal heart rate, in bpm; MCA: medial cerebral artery; UmA: umbilical artery; DV: ductus venosus. Source: authors. 
During the isometric test, subjects were qualitatively assessed to determine whether they were experiencing any pain or discomfort in the hand or forearm, muscle tremors, or an increased respiratory rate. Forty-one subjects (89.13\%) developed a tremor in the upper arm being used as they performed the isometric contractions, whereas 23 subjects (50\%) reported pain in the muscle groups of the forearm and 21 subjects (45.65\%) complained of pain only in the hand being used to grip the dynamometer. The increase in respiratory rate was observed by the ultrasonographer during the exam. This examiner noted a greater interference of maternal respiratory movements in $58 \%$ of the pregnant women as the Doppler velocimetry study of the variables was performed.

As for the fetal biometry evaluation, the estimated mean fetal weight was $2.327 \mathrm{~kg}( \pm 0.549 \mathrm{~kg})$ (range: $1.312-3.405 \mathrm{~kg})$. According to the biometry exam, 30 fetuses $(65.22 \%)$ were classified as appropriate for GA and 16 fetuses (34.78\%) were considered large for GA.

Postnatal data were obtained from 42 subjects. Of these, $80.95 \%$ had no complications, 9.52\% showed premature rupture of amniotic membranes, and $4.76 \%$ experienced premature labor. There were two cases of newborns requiring hospitalization in a neonatal intensive care unit, one of which was due to hypoxia at birth and the other was due to fetal malformation (esophageal atresia) that was not diagnosed until after birth. The mean birth weight of the newborns was $3.291( \pm 0.490) \mathrm{kg}$, with a mean GA at birth of $38.83( \pm 1.44)$ weeks. The mean first-minute Apgar score was $8.44( \pm 1.59)$, and the mean five-minute Apgar score was $9.51( \pm 1.25)$. Only two newborns were premature (Table 4$)$.

Table 4. Description of the postnatal data.

\begin{tabular}{cc}
\hline & Total $(\mathrm{N}=42)$ \\
\hline Type of birth & $24(57.14 \%)$ \\
Vaginal & $18(42.86 \%)$ \\
Cesarean section & \\
Time of birth & $40(95.24 \%)$ \\
Carried to term & $2(4.76 \%)$ \\
Preterm & \\
Apgar score & $8.44( \pm 1.59)$ \\
1 min & $9.51( \pm 1.25)$ \\
5 min & $3.291( \pm 0.490)$ \\
Average weight $(\mathrm{kg})$ & $38.83( \pm 1.44)$ \\
GA at birth in weeks & \\
Complications & $34(80.95 \%)$ \\
None & $4( \pm 9.52 \%)$ \\
Premature rupture of membranes & $2( \pm 4.76 \%)$ \\
Premature labor & $2( \pm 4.76 \%)$ \\
Need for neonatal ICU & $1( \pm 2.38 \%)$ \\
Fetal malformation &
\end{tabular}

Data represent mean \pm standard deviation or number of cases with their percentages. GA: Gestational age. ICU: intensive care unit. 


\section{Discussion}

The present study aimed to show the effects of an isometric activity protocol on maternal and fetal hemodynamics. The protocol used herein was designed for the use of a hand-held dynamometer at 50\% CVM, with isometric contractions of at least $1 \mathrm{~min}$ and with 1-min rest intervals between successive contractions.

A 2013 review states that the isometric training protocols most commonly used in research employ 30\% - 50\% CVM for at least $2 \mathrm{~min}$, with 1- to 4-min intervals between successive isometric contractions [8].

Recently, the International Working Group on Maternal Hemodynamics published recommendations for hemodynamic testing during pregnancy. In the case of the isometric test, this group recommends the use of a hand grip dynamometer at $20 \%-40 \%$ of CVM for $2-3 \mathrm{~min}$, noting that the subjects may complain of pain or discomfort and may interrupt the exercise before the time alloted [9].

Indeed, most of the subjects herein complained of hand discomfort when gripping the dynamometer, some did not engage the correct muscle groups of the upper limb in use, and some interrupted the exercise before the end of the minute or before the sonographer was able to perform the Doppler study. In these cases, the exercise and data collection steps were repeated.

It is important to note that the cross-sectional study model determined that the protocol was to be applied only once to each pregnant woman. Millar et al. commented in their review that four weeks or more of isometric training 3 - 5 times per week are required for a systemic adaptive response, with a consequent reduction in resting $B P$ [8]. However, this review sought to show the effects of isometric exercise on BP in hypertensive populations for the purposes of control and treatment of systemic arterial hypertension; this study neither included populations of pregnant women nor prioritized fetal response.

Upper-limb isometric exercise using a handgrip dynamometer was chosen because of the ease of use of the equipment, lower cost of the equipment and, most importantly, to allow access to the subject's abdomen for the ultrasound throughout the isometric exercise.

A study published in 2006 sought to determine the effects of isometric activity on BP and HR in 37 women (13 of whom were pregnant, in which case the fetal HR was also evaluated). Different loads (30\% and 50\% CVM) and different muscle groups as well as a hand grip dynamometer and a back and leg extension dynamometers were used. In their results, they reported a significant increase in maternal BP and HR and in fetal HR only when 50\% of CVM was used in the exercises involving the back and leg extension dynamometer. The same difference was not observed with the manual grip dynamometer, and it was concluded that the size of the recruited muscle mass had more of a cardiovascular impact than the intensity of the contraction [5].

In the current study, no significant hemodynamic effects were found in the fetuses; however, significant changes in maternal BP and HR were observed, which are inconsistent with the findings of other published studies. There was 
also a significant difference in the Doppler study of the left UA.

Although no significant decreases in the PI, RI, or S/D ratio values were found in the right UA during isometric testing, this artery maintained the same behavior as the left $\mathrm{UA}$, with bilateral vasodilation when maternal BP and HR were abnormal. This vasodilation mechanism of the UAs appears to be compensatory, preserving the hemodynamic homeostasis of pregnancy, a finding which is evidenced by the fact that fetal hemodynamic parameters remained unchanged.

A study published in 1993 [6] measured maternal hemodynamic response to isometric exercises of the lower limbs in 10 pregnant women (although the authors did not evaluate fetal response). They also found a significant increase in mean maternal BP and HR during exercise, with a return to the initial BP values after the rest period, but with a sustained difference in HR.

In 2000, Feiner et al. evaluated the effects of maternal isometry on placental flow in 34 pregnant women at GAs between 22 and 35 weeks using spectral Doppler. They found a significant increase in maternal parameters (systolic BP, diastolic BP, and HR) during exercise, with a return to baseline after rest; however, they did not find changes in the PI, RI, or S/D ratio values in the evaluated placental areas. Neither fetal Doppler nor fetal HR was evaluated [10].

As Meah and colleagues mentioned in their paper, few studies have reported results on fetal hemodynamics in response to isometric exercise because of the difficulty in performing fetal assessment during physical exercise; some studies have assessed the fetus shortly after the end of the exercise [9]. The fact that the fetal hemodynamic evaluation was performed at all times of the research protocol, including during the entire isometric activity performed by the pregnant women, distinguishes this study from others and is therefore one of its strengths.

It is also important to note that the entire research protocol was performed by a single interviewer, and all ultrasonographic variables were collected by the same physician using the same device, factors which reduce the chances of biases in collection or measurement.

A possible limitation of the study was the relatively short duration of the isometric exercise protocol. Isometric exercise was performed at a single instance; hence, it was not possible to evaluate long-term systemic adaptive responses in these pregnant women.

As in other epidemiological surveys and research, this study serves to reflect the alarming rate of sedentary lifestyles among women, which is even higher during pregnancy [11] [12] [13] [14]. In this study sample, $76.08 \%$ of the subjects reported being sedentary before pregnancy and $91.30 \%$ of them reported being sedentary during pregnancy. Only $8.70 \%$ of the pregnant women engaged in some kind of physical activity during pregnancy.

Studies performed in Brazil have revealed the prevalence of physically active pregnant women to be between $12.9 \%$ and $32.8 \%$ [11] [12]. In other samples, as many as $100 \%$ of pregnant women have reported being sedentary at 32 weeks of GA [13]. According to Nascimento et al., less than half of the women in their 
sample received physical activity guidelines during prenatal care [15].

In one study in the United States, $65.6 \%$ of pregnant women practiced some type of physical exercise, but only $15.8 \%$ of them were able to maintain the level of activity recommended by ACOG [16]. In a British cohort, $48.8 \%$ of pregnant women at a GA between 18 and 32 weeks had three or more hours of exercise per week [14], and in an Irish cohort, $21.5 \%$ of the pregnant women in the sample were physically active [17]. According to a study performed in Portugal, the main reasons given as a barrier to physical exercise during pregnancy were lack of time, busy schedules, and a dislike for exercise [18].

All these studies show the global issue of a sedentary lifestyle and reflect the importance of the health care professional's role in providing information on physical exercise, recommending it customarily, and knowing how to prescribe it. Given the fact that pregnant women are generally less active than adult women in the same age group, the role of the prenatal care provider becomes even more relevant; these professionals must encourage women to become and remain active throughout pregnancy in cases in which there are no contraindications.

The use of isometric exercise may facilitate pregnant women's compliance with physical activity, particularly because it is easier to perform, it does not require sophisticated equipment, and it is a shorter workout program than aerobic exercise (because it can typically be performed in half the time) [8].

\section{Conclusions}

This study was able to show significant increases in maternal HR and systolic BP during the isometric exercise protocol as well as significant decreases in the PI, $\mathrm{RI}$, and S/D ratio in the left UA during the test. In addition, the isometric test did not alter any of the fetal parameters studied.

Therefore, this study results reflect the safety of isometric exercise regarding fetal hemodynamics among low-risk healthy pregnant women.

\section{References}

[1] Department of Health and Human Services (2008) 2008 Physical Activity Guidelines for Americans. Washington, DC, DHHS.

https://health.gov/paguidelines/pdf/paguide.pdf

[2] American College of Obstetricians and Gynecologists (2015) Physical Activity and Exercise during Pregnancy and the Postpartum Period. Obstetrics \& Gynecology, 126, e135-e142.

[3] Barakat, R., Perales, M., Garatachea, N., Ruiz, J.R. and Lucia, A. (2015) Exercise during Pregnancy. A Narrative Review Asking: What Do We Know? British Journal of Sports Medicine, 49, 1377-1381.

[4] Bgeginski, R., Almada, B.P. and Kruel, L.F.M. (2015) Fetal Heart Rate Responses during Maternal Resistance Exercise: A Pilot Study. Revista Brasileira De Ginecologia E Obstetricia, 37, 133-139. https://doi.org/10.1590/SO100-720320150005132

[5] Pontes Jr., F.L., Mutarelli, M.C., Navarro, F., Moraes, M.R., de Carvalho Araújo, R. 
and Bacurau, R.F.P. (2008) Resposta cardiovascular materna e fetal ao exercício isométrico. Revista Brasileira de Ciência e Movimento, 14, 15-22.

[6] Van Hook, J.W., Gill, P., Easterling, T.R., Schmucker, B., Carlson, K. and Benedetti, T.J. (1993) The Hemodynamic Effects of Isometric Exercise during Late Normal Pregnancy. American Journal of Obstetrics \& Gynecology, 169, 870-873. https://doi.org/10.1016/0002-9378(93)90018-E

[7] Carlson, D.J., Dieberg, G., Hess, N.C., Millar, P.J. and Smart, N.A. (2014) Isometric Exercise Training for Blood Pressure Management: A Systematic Review and Meta-Analysis. Mayo Clinic Proceedings, 89, 327-334.

https://doi.org/10.1016/j.mayocp.2013.10.030

[8] Millar, P.J., McGowan, C.L., Cornelissen, V.A., Araujo, C.G. and Swaine, I.L. (2014) Evidence for the Role of Isometric Exercise Training in Reducing Blood Pressure: Potential Mechanisms and Future Directions. Sports Medicine, 44, 345-356. https://doi.org/10.1007/s40279-013-0118-x

[9] Meah, V.L., Backx, K. and Davenport, M.H. (2017) Functional Haemodynamic Testing in Pregnancy: Recommendations of the International Working Group on Maternal Haemodynamics. Ultrasound in Obstetrics \& Gynecology, 51, 331-340. https://doi.org/10.1002/uog.18890

[10] Feiner, B., Weksler, R., Ohel, G. and Degani, S. (2000) The Influence of Maternal Exercise on Placental Blood Flow Measured by Simultaneous Multigate Spectral Doppler Imaging (SM-SDI). Ultrasound in Obstetrics \& Gynecology, 15, 498-501. https://doi.org/10.1046/j.1469-0705.2000.00146.x

[11] Dumith, S.C., Domingues, M.R., Mendoza-Sassi, R.A. and Cesar, J.A. (2012) Physical Activity during Pregnancy and Its Association with Maternal and Child Health Indicators. Revista de Saúde Pública, 46, 327-333. https://doi.org/10.1590/S0034-89102012005000012

[12] Domingues, M.R. and Barros, A.J. (2007) Leisure-Time Physical Activity during Pregnancy in the 2004 Pelotas Birth Cohort Study. Revista de Saúde Pública, 41, 173-180. https://doi.org/10.1590/S0034-89102007000200002

[13] Tavares, J.D.S., Melo, A.S.D.O., Amorim, M.M.R.D., Barros, V.O., Takito, M.Y., Benício, M.H.D.A. and Cardoso, M.A.A. (2009) Padrão de atividade física entre gestantes atendidas pela estratégia saúde da família de Campina Grande-PB. Revista Brasileira de Epidemiologia, 12, 10-19. https://doi.org/10.1590/S1415-790X2009000100002

[14] Liu, J., Blair, S.N., Teng, Y., Ness, A.R., Lawlor, D.A. and Riddoch, C. (2011) Physical Activity during Pregnancy in a Prospective Cohort of British Women: Results from the Avon Longitudinal Study of Parents and Children. European Journal of Epidemiology, 26, 237-247. https://doi.org/10.1007/s10654-010-9538-1

[15] Nascimento, S.L., Surita, F.G., Godoy, A.C., Kasawara, K.T. and Morais, S.S. (2015) Physical Activity Patterns and Factors Related to Exercise during Pregnancy: A Cross Sectional Study. PLOS ONE, 10, e0133564.

https://doi.org/10.1371/journal.pone.0128953

[16] Evenson, K.R., Savitz, A. and Huston, S.L. (2004) Leisure-Time Physical Activity among Pregnant Women in the US. Paediatric and Perinatal Epidemiology, 18, 400-407. https://doi.org/10.1111/j.1365-3016.2004.00595.x

[17] Walsh, C., Farah, N., O’Dwyer, V., Hogan, J., Kennelly, M. and Turner, M.J. (2011) Maternity Services for Obese Women in Ireland. Irish Medical Journal, 104, 217-219. 
[18] Santos, P.C., Abreu, S., Moreira, C., Lopes, D., Santos, R., Alves, O., Silva, P., Montenegro, N. and Mota, J. (2014) Impact of Compliance with Different Guidelines on Physical Activity during Pregnancy and Perceived Barriers to Leisure Physical Activity. Journal of Sports Sciences, 32, 1398-1408.

https://doi.org/10.1080/02640414.2014.893369 